\title{
Prolonged-heated High-Fat Diet Increase the Serum LDL Cholesterol Level and Induce the Early Atherosclerotic Plaque Development in Wistar Rats
}

\author{
Valentina Yurina ${ }^{1 *}$, Ema Pristi Yunita ${ }^{1}$, Tri Yudani Mardining Raras ${ }^{2}$, Achmad Rudijanto ${ }^{3}$, \\ Kusworini Handono ${ }^{4}$ \\ ${ }^{1}$ Department of Pharmacy, Faculty of Medicine, Brawijaya University, Malang 65145, Indonesia \\ ${ }^{2}$ Department of Biochemistry, Faculty of Medicine, Brawijaya University, Malang 65145, Indonesia \\ ${ }^{3}$ Department of Internal Medicine, Faculty of Medicine, Brawijaya University, Malang 65145, Indonesia \\ ${ }^{4}$ Department of Clinical Patology, Faculty of Medicine, Brawijaya University, Malang 65145, Indonesia
}

Article history:

Submission May 2018

Revised July 2018

Accepted August 2018

*Corresponding author:

E-mail: v_yurina@ub.ac.id

\begin{abstract}
Rats are one of the most widely used animal models in health research. However, since rats are relatively resistant to atherosclerosis, the transgenic rats often used to study atherosclerosis in rats. Our study suggests a prolonged-heated lipid to induce atherosclerosis in rats, therefore provide a more low-cost option to study atherosclerosis in rats. Aims of this study is to compare the effect of heated highfat diet (HFD) to the LDL and HDL cholesterol level and foam cell formation in the Wistar rat animal model. Rats were divided into three groups, control group was given with the normal diet, and the other two treated groups received oxidized HFD (heated HFD) and HFD, respectively. The heated HFD contain lard that was previously heated at $190^{\circ} \mathrm{C}$ for $24 \mathrm{~h}$. Diet was given for 8 weeks. The serum LDL and HDL cholesterol level were measured before and $4^{\text {th }}$ and $8^{\text {th }}$ weeks after treatment with help of colorimetric measurement. The aorta analyzed for the foam cell formation after HE staining using the light microscope. The prolonged- heated HFD significantly increased the LDL cholesterol from the $4^{\text {th }}$ week of the treatment $(p=0.023)$. The rats aortic from HFD and heated HFD treated groups showed a mononuclear cells infiltration and the early foam cell formation. The heating process of the lipid caused lipid oxidation which significantly increased the LDL cholesterol level of the animal model and foam cells formation. This study suggests that Wistar rats with heated HFD could demonstrated early atherosclerosis plaque development.
\end{abstract}

Keywords: Cholesterol, foam cells, high-fat diet (HFD), oxidized lipid, atherosclerosis

\section{Introduction}

Nowadays, cardiovascular disease, including coronary heart disease, stroke, cardiomyopathy, and other diseases, is counted as the major cause of mortality in the world [1]. Although the incidence tends to decrease in the developed countries, the tendency is rising in the low and middleincome countries. The lifestyle changes, including high fat intake, less physical activity, and smoking habits take part in the increased cardiovascular disease prevalence [1, 2].

Atherosclerosis is the chronic inflammatory disease which causes the cardiovascular disease $[3,4]$. One of the most important risk factors of the atherosclerosis is dyslipidaemia which is characterized by the high concentration of total cholesterol, triglyceride, and LDL cholesterol, and low concentration of HDL cholesterol in the blood [5, 6]. Moreover, the radical oxygen species (ROS) enhance the production of the oxidized LDL cholesterol which initiates the progression of atherosclerosis [7].

Oxidized lipid binds to the scavenger receptor, such as lectin-like oxidized-LDL receptor-1 
(LOX-1), in the endothelial artery cells and activate the endothelial dysfunction [8, 9]. Subsequently, the activated endothelial cells release cytokine and adhesion molecules which promote the monocytes recruitment that differentiate into macrophages [10] and generate the foam cells [11] which develops to atherosclerotic plaques [12, 7 , 4].

To study atherosclerosis, some animals' models have been used. Rats are among the most favourable animal model because it is low cost, easy to handling and housing, and well-establish genomic data [13]. Although these animal models have important roles in understanding the atherosclerosis lesion formation, there are some limitations of using these animal [14]. Rats are relatively resistant to atherosclerosis because most of the total plasma cholesterol is in the HDL form. In addition, rats also do not have cholesteryl ester transfer protein (CETP) which make them more unsusceptible for atherosclerosis [15]. Several transgenic rats have been developed to study atherosclerosis, such as the CETP transgenic rats [13, 15]. However, the transgenic animals are highly cost and not widely available. Aims of this study were to investigate the effect of prolong-heated HFD to the cholesterols levels in the animal models. Moreover, we also examine the effect of the diet to the formation of the foam cells in the aorta and the fatty liver development. The prolongedheated affects the chemical and physical changes in the lipid composition and structure. The heating process increases the oxidation, hydrolysis, and polymerization, and changing of the fatty acid from cis to trans isomers [16]. The research offers a low-cost option to develop rats as the atherosclerosis model.

\section{Material and Methods \\ Animal treatment}

Male Wistar rats (Rattus norvegicus) $(\mathrm{n}=15)$ were allowed for acclimatization for one week prior to treatment. They were randomly divided into three groups, the control group was given normal diet, the treated groups were fed high-fat diet (HFD), and heated high-fat diet (heated HFD) subsequently. The diets were given for eight weeks. The diet composition is described in Table 1. The high-fat diets were prepared by adding $2 \%$ cholesterol powder, $0.3 \%$ cholic acid, and $10 \%$ lard [17]. The heated HFD was prepared by heating the diet at $190^{\circ} \mathrm{C}$ for $24 \mathrm{~h}$ [12]. The diets were made weekly and kept in the refrigerator until used. Each animal was given $20 \mathrm{~g}$ of diets daily, based on their groups. The ingested feed was measured daily. Drinking water was given ad libitum throughout the experiment. Body weight was measured weekly. Serum from blood was obtained from tail vein prior treatment and after 4 th and $8^{\text {th }}$ weeks of the administration using high fat diet. After 8 weeks, the rats were sacrificed and the aortas and the livers were collected.

\section{Foam cell and liver histopathology analysis}

Aortas were fixed and embedded with paraffin. Specimens were sliced and stained with Haematoxylin Eosin using the conventional methods. Histological analysis was performed under 400× magnification light microscope (Olympus, Tokyo, Japan). Livers were excised from the animals, rinsed with PBS, and physically examined.

\section{Statistical analysis}

All values were revealed as means $\pm \mathrm{SD}$. Collected data were analyzed using one-way ANOVA and post-hoc LSD Test and the results were considered significant at $\mathrm{p}<0.05$.

\section{Results and Discussion \\ The HFD and heated HFD diet increases the LDL cholesterol serum level}

The HFD and heated HFD diet successfully increased the LDL cholesterol level in serum. After 4 weeks' treatment with HFD diet, the LDL cholesterol serum levels in the groups received HFD

Table 1. Diet composition

\begin{tabular}{lccc}
\hline \multirow{2}{*}{ Composition } & \multicolumn{3}{c}{ Percentage } \\
\cline { 2 - 4 } & $\begin{array}{c}\text { Normal } \\
\text { Diet }\end{array}$ & $\begin{array}{c}\text { High Fat } \\
\text { Diet }\end{array}$ & $\begin{array}{c}\text { Heated High } \\
\text { Fat Diet }\end{array}$ \\
\hline Cornstarch & 55 & 42.7 & 42.7 \\
Soybean oil & 7 & 7 & 7 \\
Casein & 20 & 20 & 20 \\
Sucrose & 10 & 10 & 10 \\
Cellulose & 5 & 5 & 5 \\
Gelatin & 3 & 3 & 3 \\
Lard & 0 & 10 & $10 *$ \\
Cholic acid & 0 & 0.3 & 0.3 \\
Cholesterol & 0 & 2 & 2 \\
Total & 100 & 100 & 100 \\
\hline
\end{tabular}

* heated for $24 \mathrm{~h}, 190^{\circ} \mathrm{C}$ 
and heated HFD were higher than groups fed with the normal diet. Furthermore, after 8 weeks of the diet, the serum LDL cholesterol in both groups were higher than the LDL cholesterol level in the normal group diet (Figure 1A). Surprisingly, the diet also increased the HDL serum level in the all groups. However, after 8 weeks the HDL cholesterol serum level declined in all groups (Figure 1B). The groups received HFD had the lowest serum HDL cholesterol level among the three groups, measurement of the body weights of all animals weekly showed that after 8 weeks of treatment with HFD diet, there was no significant differences were observed among all groups (data not shown). The normal groups demonstrated the most increased body weight because they also had the most ingested food (data not shown).

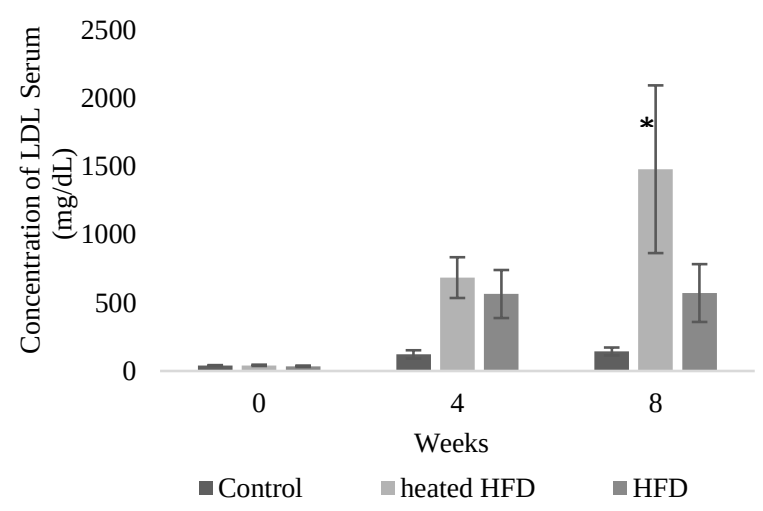

(a)

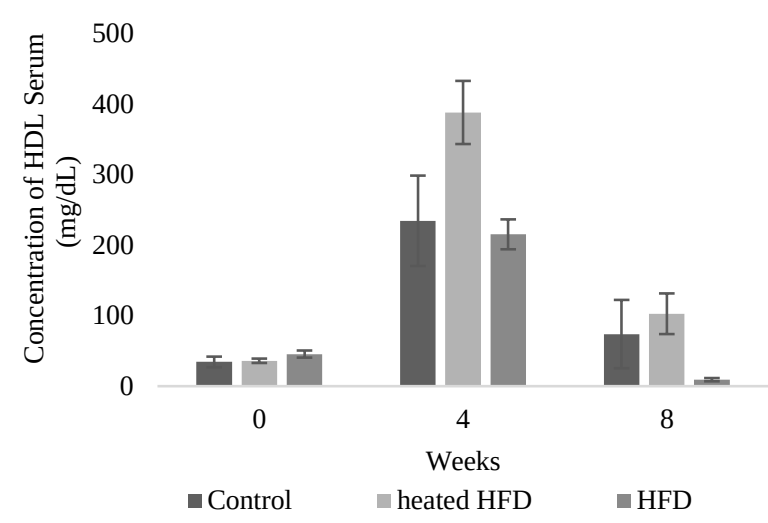

(b)

Figure 1. The serum LDL (a) and HDL (b) cholesterol level analysis results. Analysis showed that the heated HFD diets significantly increase the LDL cholesterol level after 8 weeks' treatment $(\mathrm{p}$ value $=0.023)$. Notes: $*$ shows p value $<0.05$ against normal diet.
High consumption of unhealthy fats and oil lead to the pathogenesis of dyslipidaemia, a major risk factor for atherosclerosis and cardiovascular diseases $[18,1]$. Not only are the excessive consumption of lipid, to reduce cost, the frying oils repeatedly used in many communities, especially in the low income or middle-income countries. The prolong or repeatedly heating process increases the free radicals' level in animal study [19]. Other study demonstrated that higher temperature and longer heating process lead to the increased lipid peroxidation level in rats. The thermal process directs to the polyunsaturated fatty acid decomposition and other fatty acid composition changes [12]. Our study indicated the similar result, where the group received thermally heated HFD had higher LDL cholesterol level than the LDL level of the group receive HFD diet after 8 weeks of treatment. While the LDL cholesterol level of the groups received normal diet is significantly lower than that of group received heated HFD diet.

In the first 4 weeks, the HDL cholesterol level in all groups rose substantially with highest level was obtained by the group with the heated HFD HDL. This phenomenon demonstrated the reverse cholesterol transport in which the HDL plays a major role in cholesterol efflux [20]. Reverse cholesterol efflux (RCT) is a process in which the cholesterol is effluxed from blood and returned to the liver by the HDL. Furthermore, the cholesterol is excreted into bile and faeces. However, dyslipidaemia has been proven to impair this process through lowering the HDL level [21]. Our result showed a similar trend i.e. after 8 weeks of treatment, the HDL level of all treated groups was decreased. The group received heated HFD had the most increasing HDL level after 4 weeks (10.78fold increasing). This result suggested that the heated HFD diet induced RCT process to overcome the high amount of cholesterol intake. However, the group received HFD only demonstrated the lowest g HDL level after 8 weeks of treatment (23.33-fold decreasing) suggested that in this groups the RCT process was the most impaired.

\section{HFD and heated HFD diet generates the foam cell formation}

Examination of aortic region of the normal rats showed a normal character with the smooth endo- 
A.
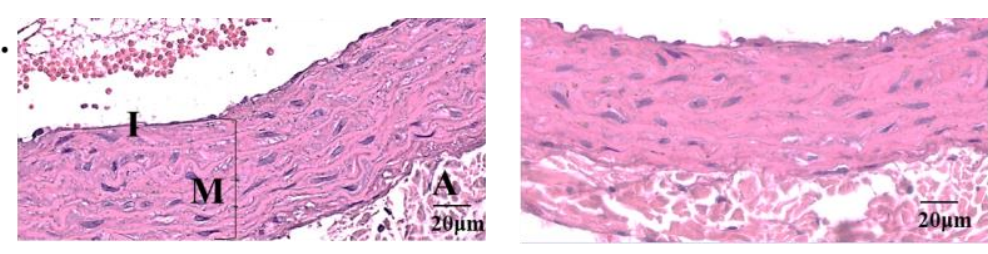

B.
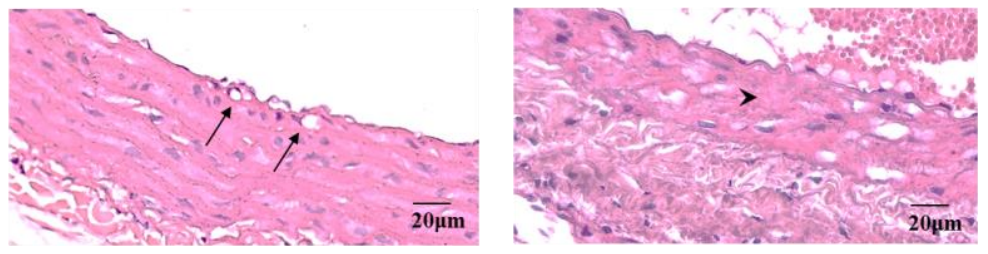

C.
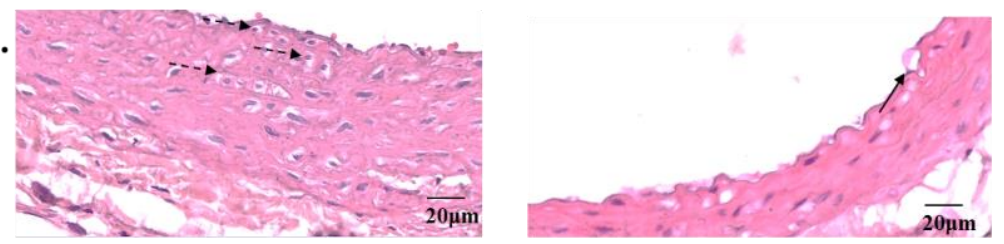

Figure 2. Light microscopic analysis of HE stained aorta section after eight weeks' treatment with normal diet (A), heated HFD (B), and HFD (C), (magnification 200×). Each groups were represented with 2 aortic regions from different animals. Rats with normal diet showed thin and smooth vessels. Smooth muscle cells in the tunica media oriented horizontal to the aortic canal (A). In the rats with HFD, mononuclear infiltration cells appeared in the media (dashed arrows) (C); the foam cells also observed under the sub- intima (arrows) (B). Some area also showed losing of muscle cells in the heated HFD groups (arrow heads) (B). Notes: $\mathrm{I}$ = intima, $\mathrm{M}=$ media, $\mathrm{A}=$ adventitia
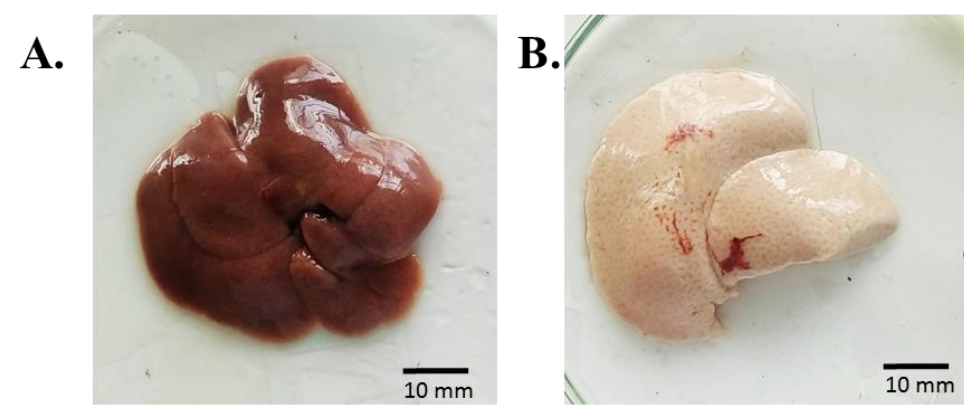

Figure 3. Liver gross examination showed that heated HFD induced fat accumulation. Liver from normal diet rat showed a normal, red, and shiny appearance (A), while the heated HFD diet induced fatty liver like phenomena that is indicated by extremely pale appearance of the liver (B).

thelial cells. The muscle cells in the tunica media were observed in horizontal direction while the collagen and the connective tissues were also normal. After eight weeks' administration with heated HFD, accumulation of fatty cells was found in the sub-intima of the aorta. The mononuclear cells infiltration was also observed in both groups. Some areas in the aortic of group with heated HFD demonstrated losing of muscle cells (Figure 2).

The HFD affects the LDL cholesterol level in the blood and its vulnerability to the oxidation process. Lipid oxidation acts as an initiation of the en- dothelial dysfunction leads to the foam cell formation and atherosclerosis plaque development [22]. HFD together with the cholesterol supplementation in the diet has the pro-atherogenic effect [23]. The aortic staining results revealed that the HFD altered the endothelial cells morphology after 8 weeks of the diet. Both groups received HFD diets showed a mononuclear cells infiltration in the intima-media regions. The mononuclear cells, such as phagocytes and monocytes, recruitment indicates an early stage of atherosclerosis [24]. Later on, the recruited monocytes undergo a mod- 
ification and maturation to macrophage. The uptake of the ox-LDL in the macrophages results in the foam cell formations which develop the atherosclerosis plaque [25]. Foam cells were found in the aortic sub-intima region from both groups. In some part of the aortic regions of the heated HFD diet rats, the smooth muscle cells disorientation and apoptosis were also observed (Figure 2). The smooth muscle cells apoptosis generally observed in the advanced lesion of the atherosclerotic plaque [26]. However, previous studies also indicated that smooth muscle cells apoptosis has been associated with the progression of the inflammation process, calcification, and plaque rupture [2628]. On the other hand, the rats fed the normal diet showed a normal layer of endothelial cells in thin and smooth layers.

\section{Heated HFD induces the fat accumulation in liver}

Macroscopic analysis of normal diet rats showed a normal, red, and shiny liver. In contrast, the rats with heated HFD diets showed an extremely pale appearance that indicates a phenomena-like fatty liver (Figure 3).

Liver gross analysis revealed that the heated HFD promotes the fat accumulation in the liver that is shown by the extremely pale appearance of the organ. The cholesterol and cholic acid in the diet have been studied to in inducing the liver steatosis, necrosis, hepatocyte ballooning, steatohepatitis and fibrosis in the animal model [29]. This results also suggest that the heated HFD can lead to the development of non-alcoholic fatty liver disease (NAFLD), one of the most common chronic liver diseases in the Western world [30]. NAFLD in human is developed after years and caused by several risk factors, such as unhealthy diet, less physical activity, stress, and lifestyle. It is also known as a polygenic disease in which many genes take part to influence the condition. However, the high fat diet which leads to dyslipidemia, obesity, and metabolic disease is considered as a major risk factor [29]. Our result revealed the similar phenomenon, in which after 8 weeks of HFD, the liver analysis showed a very clear pathological condition related to fat accumulation in the liver.

Our result suggest that the prolonged-heated high fat diet can be applied in developing animal model for studying atherosclerosis in rats. As widely accepted, rat is resistant to atherosclerosis and it is considered as an unsuitable animal for studying lipid accumulation and atherosclerosis. Rat's cholesterol is in the HDL form and rat do not have the CETP [15]. Although several strains of transgenic rats are available for studying atherosclerosis, however, these transgenic rats usually are expensive. Thus, our study offers an alternative diet that is economical and easily managed which can induce early atherosclerosis lesions. The result indicated that heated HFD significantly increased the LDL cholesterol level and developed early stage of atherosclerosis. Thus, the prolonged-heated HFD provides an option to work with Wistar rats as atherosclerosis animal model.

\section{Conclusion}

Our study suggests rats fed with prolonged heated HFD had a higher LDL cholesterol level than rats with HFD only. Both groups received HFD and heated HFD demonstrated mononuclear cells infiltration, muscle cells degeneration, and foam cells formation in its aortic area. Heated HFD induced the fatty liver accumulation after eight weeks of treatment. Hence, Wistar rats with prolonged-heated HFD can describe the early atherosclerosis lesion.

\section{Acknowledgment}

We would like to show our gratitude to Adhika Twas Galih Atyanta, Mochtaromi Tri Yanto, Elan Aisyafuri, Dina Sulastiyo Murti, Yanti M. Parhusip, and Arinal Muna Al-Muyasaroh for their help during the animal treatment.

\section{References}

1. van der Ende MY, Hartman MHT, Hagemeijer Y et al. (2017)

The lifelines cohort study: Prevalence and treatment of cardiovascular disease and risk factors. International Journal of Cardiology 228495 - 500. doi: 10.1016/j.ijcard.2016.11.061.

2. Lloyd-Jones DM, Leip EP, Larson MG et al. (2006) Prediction of lifetime risk for cardiovascular disease by risk factor burden at 50 years of age. Circulation 113: 791-798. doi: 10.1161/CIRCULATIONAHA.105.548206.

3. Libby P (2003) Vascular biology of atherosclerosis: Overview and state of the art. The American journal of cardiology 91 (2): 3A - 6A. doi: 10.1016/S0002-9149(02)03143-0.

4. Frostegård J (2013) Immunity, atherosclerosis and cardiovascular disease. BMC Medicine 11: 117. doi: 10.1186/1741-701511-117.

5. Vaziri ND (2014) Role of dyslipidemia in impairment of energy metabolism, oxidative stress, inflammation and cardiovascular 
disease in chronic kidney disease. Clinical and Experimental Nephrology 18 (2): 265 - 268. doi: 10.1007/s10157-013-0847Z.

6. Gami AS, Witt BJ, Howard DE et al. (2007) Metabolic syndrome and risk of incident cardiovascular events and death: A systematic review and meta-analysis of longitudinal studies. Journal of the American College of Cardiology 49 (4): 403-414. doi: 10.1016/j.jacc.2006.09.032.

7. Bonomini F, Tengattini S, Fabiano A et al. (2008) Atherosclerosis and oxidative stress. Histology and Histopathology 23 (3): 381-390. doi: 10.14670/HH-23.381.

8. Moriwaki H, Kume N, Sawamura T et al. (1998) Ligand specificity of LOX-1, a novel endothelial receptor for oxidized low density lipoprotein. Arteriosclerosis, Thrombosis, and Vascular Biology 18 (10): 1541 - 1547. doi: 10.1161/01.ATV.18.10.15 41.

9. Sugiyama D, Higashiyama A, Wakabayashi I et al. (2015) The relationship between lectin-like oxidized low-density lipoprotein receptor-1 ligands containing apolipoprotein B and the cardio-ankle vascular index in healthy community inhabitants: The KOBE study. Journal of Atherosclerosis and Thrombosis 22 (5): 499 - 508. doi: 10.5551/jat.26450

10. Mango R, Predazzi IM, Romeo F, Novelli G (2011) LOX1/LOXIN: The yin/yang of atheroscleorosis. Cardiovascular drugs and therapy / sponsored by the International Society of Cardiovascular Pharmacotherapy 25 (5): 489 - 494. doi 10.1007/s10557-011-6333-5.

11. Rader DJ, Puré E (2005) Lipoproteins, macrophage function, and atherosclerosis: Beyond the foam cell? Cell Metabolism 1 (4): 223 - 230. doi: 10.1016/j.cmet.2005.03.005.

12. Eder K, Keller U, Hirche F, Brandsch C (2003) Thermally oxidized dietary fats increase the susceptibility of rat LDL to lipid peroxidation but not their uptake by macrophages. The Journal of Nutrition 133 (9): 2830 - 2837. doi: 10.1093/jn/133.9.2830.

13. Duelsner A, Persson AB (2013) Animal models in cardiovascular research. Acta Physiologica 208 (1): 1 - 5. doi: 10.1111/ apha.12074

14. Kapourchali FR (2014) Animal models of atherosclerosis. World Journal of Clinical Cases 2 (5): 126 - 132. doi 10.12998/wjcc.v2.i5.126.

15. Xiangdong L, Yuanwu L, Hua Z et al. (2011) Animal models for the atherosclerosis research: A review. Protein and Cell 2 (3): 189 - 201. doi: 10.1007/s13238-011-1016-3.

16. Nawar WW (1984) Chemical changes in lipids produced by thermal processing. Journal of Chemical Education 61 (4): 299 - 302. doi: 10.1021/ed061p299.

17. Riahi S, Mohammadi MT, Sobhani V et al. (2015) Chronic effects of aerobic exercise on gene expression of LOX-1 receptor in the heart of rats fed with high fat diet. Iranian Journal of Basic Medical Sciences 18 (8): 805 - 812.

18. Fruchart JC, Nierman MC, Stroes ESG et al. (2004) New risk factors for atherosclerosis and patient risk assessment. Circulation 109: III15 - I19. doi: 10.1161/01.CIR.0000131513.33892. $5 \mathrm{~b}$.

19. Venkata RM, Subramanyam R (2016) Evaluation of the deleterious health effects of consumption of repeatedly heated vegetable oil. Toxicology Reports 3: 636 - 643. doi: 10.1016/j.toxrep.2016.08.003.

20. Escolà-Gil JC, Llaverias G, Julve J et al. (2011) The cholesterol content of western diets plays a major role in the paradoxical increase in high-density lipoprotein cholesterol and upregulates the macrophage reverse cholesterol transport pathway. Arteriosclerosis, Thrombosis, and Vascular Biology 31 (11): 24932499. doi: 10.1161/ATVBAHA.111.236075.

21. Tréguier M, Briand F, Boubacar A et al. (2011) Diet-induced dyslipidemia impairs reverse cholesterol transport in hamsters. European Journal of Clinical Investigation 41 (9): 921 - 928. doi: 10.1111/j.1365-2362.2011.02478.x.

22. Libby P, Ridker PM, Hansson GK (2011) Progress and challenges in translating the biology of atherosclerosis. Nature 473: 317 - 325. doi: 10.1038/nature10146.

23. Khan-Merchant N, Penumetcha M, Meilhac O, Parthasarathy S (2002) Oxidized fatty acids promote atherosclerosis only in the presence of dietary cholesterol in low-density lipoprotein receptor knockout mice. The Journal of nutrition 132 (11): 3256 3262. doi: 10.1093/jn/132.11.3256

24. Libby P, Ridker PM, Hansson GK (2009) Inflammation in atherosclerosis from pathophysiology to practice. Journal of the American College of Cardiology 54 (23): 2129 - 2138. doi: 10.1016/j.jacc.2009.09.009.

25. Weber C, Noels H (2011) Atherosclerosis: current pathogenesis and therapeutic options. Nature Medicine 17 (11): 1410 - 1422. doi: 10.1038/nm.2538.

26. Clarke M, Bennett M (2006) The emerging role of vascular smooth muscle cell apoptosis in atherosclerosis and plaque stability. American journal of nephrology 26 (6): 531-535. doi: 10.1159/000097815.

27. Ishino S, Mukai T, Kume N et al. (2007) Lectin-like oxidized LDL receptor-1 (LOX-1 ) expression is associated with atherosclerotic plaque instability - analysis in hypercholesterolemic rabbits. Atherosclerosis 195 (1): 48 - 56. doi: 10.1016/j.atherosclerosis.2006.11.031.

28. Rudijanto A (2007) The role of vascular smooth muscle cells on the pathogenesis of atherosclerosis. Acta Medica Indonesiana 39 (2): 86 - 93

29. Kucera O, Cervinkova Z (2014) Experimental models of nonalcoholic fatty liver disease in rats. World Journal of Gastroenterology 20 (26): 8364 - 8376. doi: 10.3748/wjg.v20.i26.8364.

30. Van Herck MA, Vonghia L, Francque SM (2017) Animal models of nonalcoholic fatty liver disease-a starter's guide. Nutrients 9 (10): 1 - 13. doi: 10.3390/nu9101072. 